\title{
Anomalous Facial Nerve: An Unusual Cause of Obstruction of Middle Ear Ventilation
}

\section{Das S, Tuli I P}

Department of ENT

Sikkim Manipal Institute of Medical Science

Tadong, Sikkim - 737102, India

Corresponding Author

Soumyajit Das

Department of ENT

Sikkim Manipal Institute of Medical Science

Tadong, Sikkim - 737102, India

Email: drsoumya_entamch@rediffmail.com

\section{Citation}

Das S, Tuli IP. Anomalous Facial Nerve: An Unusual Cause of Obstruction of Middle Ear Ventilation. Kathmandu Univ Med J 2015;49(1):74-6.

\begin{abstract}
Numerous anomalies and variations of facial nerve anatomy leading to iatrogenic injury are described. However, there are no reports of facial nerve dehiscence near its second genu causing a hump and obstructing middle ear ventilation pathway, as found in our case.

This particular anomaly of facial nerve is being reported to highlight its uniqueness and that a dehiscent facial nerve may be a rare but dangerous cause of obstruction of the attic ventilation. One has to be aware of this unusual anomaly to prevent inadvertent damage to the facial nerve while clearing aditus block in persistent otitis media.
\end{abstract}

\section{KEY WORDS}

Facial nerve, facial nerve anomaly, facial nerve dehiscence.

\section{INTRODUCTION}

The tortuous and long intra-osseous course of the facial nerve makes it unique with respect to other cranial nerves as well as vulnerable to intra-operative insult. While the anomalies of the facial nerve are commonly found in association with various congenital malformations of the ear, they may be found in apparently in normal ears. Such unexpected abnormal positions encountered during the surgery can jeopardize the integrity of the nerve and land the surgeon in trouble. ${ }^{1}$

\section{CASE-REPORT}

A 26 year old male, presented with recurrent episodes of profuse mucoid discharge from the left ear for 15 years that was neither foul smelling nor blood stained. The patient never achieved a completely dry ear, despite prolonged conservative treatment. He also gave a history of gradual onset non-progressive hearing loss in his left ear for the past 15 years. There was no history of ear ache, tinnitus, vertigo or any history suggestive of complicated Otitis media. On examination, there was a subtotal perforation of the tympanic membrane (TM) with edematous middle ear mucosa on the left ear. There was no evidence of any facial weakness. Pure tone average on Pure tone audiometry (PTA) showed conductive loss of $45 \mathrm{dBHL}$ There was no disease in the sinonasal cavity. Plain radiograph of the mastoids showed sclerosed mastoid with an intact and normal lying tegmen and the sinus plate. General, systemic examination, routine blood examination, chest $X$ ray and kidney function tests were normal. A diagnosis of Otitis media, Active mucosal type was made and Cortical mastoidectomy with Type I tympanoplasty on the left ear under general anesthesia was done using post auricular approach. Intraoperatively, the mucosa inside the antrum was edematous and the attic ventilation pathway was completely blocked by the anomalous position of the facial nerve. The facial nerve was dehiscent from the $2^{\text {nd }}$ genu onwards and was also blocking the ventilation pathway of the attic by a hump formation. (Fig. 1). The water test was negative. The ossicular chain was intact. 


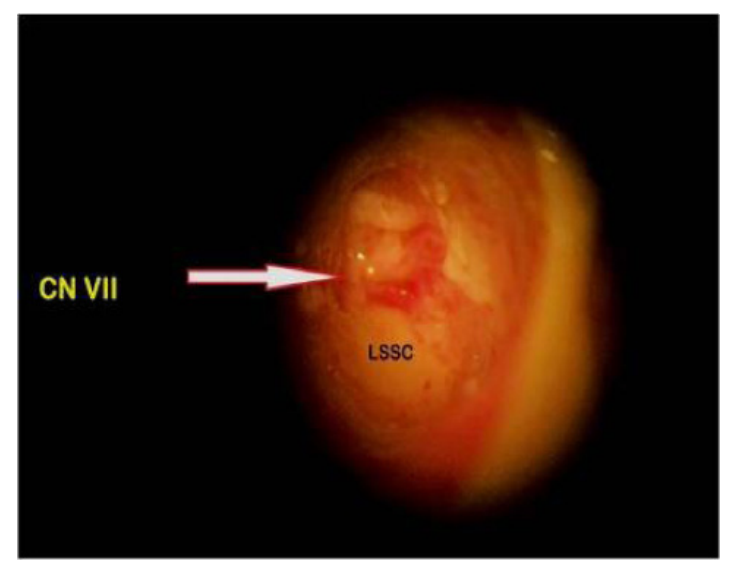

Figure 1. Dehiscent Facial nerve blocking the attic, 1.6X Opto Microscope. LSCC - lateral semicircular canal.

The edematous mucosa around the attic and the exposed facial nerve was carefully removed. The attic widened enough to allow free flow of water. After ensuring the ossicular chain mobility, temporalis fascia graft was inserted by underlay technique.

The facial nerve function was normal post operatively and the patient had an uneventful recovery. On follow up at the end of 6 weeks the temporal is graft was found to be in situ.

\section{DISCUSSION}

Chronic mastoiditis is a common indication for cortical mastoidectomy and very often per-operatively, the attic ventilation pathway is found to be obstructed by edematous mucosa or granulation tissue. The outcome of this surgery depends on establishing the ventilation pathways in the middle ear. There are three pathways providing ventilation of the middle ear and the mastoid cavity. The anterior pathway lies medial to the anterior malleolar ligament and chorda tympani canal, anterior to tensor tympani tendon. The middle pathway lies medial to the malleus- incus complex and lateral to the facial canal, posterior to the cochleariform process and tensor tympani tendon and anterior to stapes superstructure. The posterior pathway lies medial to the body and short process of the incus and lateral to facial canal, posterior to stapes superstructure and anterior to fossa incudis and posterior canal wall. Unless these pathways are established properly there is high chance of failure of the surgery. ${ }^{2}$

The facial nerve often shows anatomical abnormalities in its intra-temporal course. These abnormalities are common in patients with developmental malformations of the ear, yet may be found in a normal temporal bone also. Congenital bony dehiscences in the facial canal result from incomplete closure during development and are observed in approximately 55\% of temporal bones. ${ }^{3}$ The most common abnormality that is encountered is the dehiscence of the Fallopian canal and in most cases this dehiscence lies in the tympanic segment.
Baxter found that $57 \%$ of people have dehiscence of the facial canal in the oval niche. ${ }^{4}$ Takahashi and Sando reported facial canal dehiscence in $74 \%$ temporal bones and the most frequent site of dehiscence was at the oval window. ${ }^{5}$ Moreano et al. examined 1000 temporal bones and found unilateral dehiscence of facial canal in $56 \%$ and bilateral dehiscence in $76.3 \%$ of temporal bones. ${ }^{6}$ Temporal bone specimen of newborns revealed much higher incidence of multiple dehiscence along the facial canal. ${ }^{7}$ Di Martino et al. reported a dehiscence rate of $6.4 \%$ in his series of 357 operated cases. ${ }^{8}$ Yetiser, in his study on 144 cases of middle ear surgery concluded that approximately one out of every 10 cases undergoing mastoid surgery had dehiscence in the fallopian canal. ${ }^{1}$ Ballester et al in their series of 595 stapedotomies described an abnormal course of facial nerve lying over the oval window. ${ }^{9}$ The dehiscent facial nerve with prolapse over the oval window may partially or completely obliterate the stapes foot plate causing surgical challenge even to the experienced otologists. The dehiscent facial nerve is susceptible to injury if care is not taken during surgery.

The region of the second genu is a common site for iatrogenic injury. Yadav et al have reported a lateral hump at the second genu or posterolateral bulge below the horizontal semi circular canal in $20 \%$ temporal bone specimens. In the same study the $2^{\text {nd }}$ genu was lateral to the prominence of lateral semicircular canal in $12 \%$ specimens. ${ }^{10}$ Lateral hump anomaly of the pyramid segment has also been reported in $4.83 \%$ of cases undergoing middle ear surgery. ${ }^{11}$ The lateral semicircular canal (LSCC) serves as a safe landmark to identify and protect the facial nerve, which is inferomedial to the LSCC. However, the presence of a laterally located facial nerve can confound the surgeon and iatrogenic injury may occur.

Other rare anomalies of the facial nerve that have been reported include its duplication often associated with middle and inner ear anomalies, bifurcation of tympanic segment seen with congenital stapes fixation and oval window atresia and bifurcation of the labyrinthine segment,commonly associated with cochlear and vestibular anomalies. ${ }^{12,13}$ Bifurcation of intracanalicular part of the facial nerve with separate or no bony septa has also been described. ${ }^{12,13}$ Such duplications and bifurcations may lead to inadvertent iatrogenic injury of the unidentified portion of the facial nerve if the surgeon is unaware of this anomaly.

In patients with external auditory canal atresia, the facial nerve often shows altered course within the mastoid segment passing along or through the atretic bone plate resulting in increased risk of injury during surgical repair. Anterolateral displacement of stylomastoid foramen may make the nerve vulnerable to iatrogenic injury. ${ }^{14}$

The facial nerve in the present case was found dehiscent with an unexpected hump formation. The dehiscence extended from the $2^{\text {nd }}$ genu towards the tympanic segment. The dehiscent segment with the hump was lying in the 
attic region and blocking the aditus and was covered by the edematous mucosa of the antrum and aditus impeding the normal ventilation pathway. We feel that this particular situation was unique in its presentation for two reasons. Obstruction of ventilation pathway of the attic mostly occurs due to edematous mucosa or granulation tissue, but a dehiscent facial nerve with a hump causing the same has not been reported so far. Secondly, the precarious position of the facial nerve in the attic region makes it vulnerable to accidental injury at the time of widening the attic ventilation pathway. One might be tempted to undergo pre operative HRCT of the mastoids in such cases of safe disease to predict such anomaly. However we feel that such an expensive modality of investigation is not justified as it is not a common scenario. A little caution on the part of the surgeon and a knowledge of the anomaly can prevent injury to the nerve. Though use of diamond burr is advisable rather than cutting burr for widening the attic in such situation, we preferred using a curette for widening the bone in the attic to prevent injury by unintentional slipping of the burr. Any soft tissue blocking the aditus must not be blindly dissected or pulled, without ascertaining that it is not a part of the facial nerve. Routine use of antrum seeker, ball probe, sickle knife or Rosen's needle in the aditus area to ascertain the position of the aditus is also to be condemned.
Anomalies of the facial nerve are mostly associated with inner and middle ear abnormalities. It is also important to note that the facial nerve may also run an anomalous course in absence of other abnormalities of the middle or the inner ear and may occur sporadically. ${ }^{15}$ An otologic surgeon must have a thorough knowledge of anatomy to anticipate such anomalies in order to prevent inadvertent damage to the facial nerve.

\section{CONCLUSION}

Various anatomical abnormalities of the facial nerve are described. However, we have not found any report of a dehiscent facial nerve causing attic obstruction and thus persistent ear disease as has been seen in this case, despite extensive literature search. This case is reported for its unique presentation and to emphasize that an otologic surgeon has to be aware of anatomical abnormalities of the facial nerve that may be encountered unexpectedly during the course of surgery. Any thick white cord like structure within the middle ear cavity or even in the aditus has to be dealt with care and considered to be facial nerve until proven otherwise. In such cases the surgeon should always establish the landmarks of the facial nerve and identify the facial nerve beyond doubt before severing any structures.

\section{REFERENCES}

1. Yetiser S. The dehiscent facial nerve canal. Int J Otolaryngol. 2012(2012). http://dx.doi.org/10.1155/2012/6797089 (accessed on $16^{\text {th }}$ September 2013)

2. Mahadevaiah A, Parikh B. Surgical techniques in chronic otitis media and otosclerosis. $2^{\text {nd }}$ ed. N.Delhi:CBS publishers; 2011.

3. Nager GT, Proctor B. Anatomic variations and anomalies involving the facial canal. Otolaryngol Clin North Am 1991 Jun;24(3):531-53.

4. Baxter A. Dehiscence of the fallopian canal. J Laryngol Otol 1971;85(6):587-94

5. Takahashi H, Sando I. Facial canal dehiscence: histologic study and computer reconstruction. Ann Otol Rhinol Laryngol 1992:101(11):925-30.

6. Moreano EH, Paparella MM, Zelterman D, Goycoolea MV. Prevalence of facial canal dehiscence and of persistent stapedial artery in the human middle ear: a report of 1000 temporal bones. Laryngoscope 1994;104(3, part 1):309-20.

7. Perez B, Campos ME, Rivero J, Campos DL, López-Aguado D. Incidence of dehiscence in the fallopian canal. Int J Pediatr Otorhinolaryngol 1997;40(1):51-60.

8. Di Martino E, Sellhaus B, Haensel J, Schlegel JG, Westhofen M, Prescher A. Fallopian canal dehiscence: a survey of clinical and anatomical findings. Eur Arch Otorhinolaryngol 2005:262(2):120-6.

9. Ballester M, Blaser B, Häusler R. Stapedotomy and anatomical variations of the facial nerve. Rev Laryngol Otol Rhinol 2000:121(3):181-6.

10. Yadav SPS, Ranga A, Sirohiwal BL, Chanda R. Surgical anatomy of tympano-mastoid segment of facial nerve. Indian J Otolaryngol Head Neck Surg 2006:58(1): 27-30.

11. Dai C, Li J, Zhao L, Liu Y, Li Y, Song Z, Feng S, Lu J. Lateral hump anomaly of pyramid segment of facial nerve in living patients. Acta otolaryngol 2013:133(9): 1006-8.

12. Glastonbury CM, Fischbein NJ, Harnsberger HR, Dillon W P, Kertesz TR. Congenital bifurcation of the intratemporal facial nerve. AJNR Am J Neuroradiol 2003;24(7): 1334-37.

13. Celin SE, Wilberger JE, Chen DA. Facial nerve bifurcation within the internal auditory canal. Otolaryngol Head Neck Surg 1991;104(3): 389-93.

14. Yeakley JW, \& Jahrsdoerfer RA. CT evaluation of congenital aural atresia: what the radiologist and surgeon need to know. J Comput Assist Tomog 1996;20(5):724-31.

15. Tange RA, De Bruijn AJG. Dehiscences of the horizontal segment of the facial canal in otosclerosis. ORL J Otorhinolaryngol Relat Spe 1997:59(5): 277-9. 